\section{Transpupillary thermotherapy for treatment of exudative age- related macular degeneration in Japanese patients}

T Atarashi, Y Tamaki, Y Inoue, R Obata, $K$ Muranaka and $Y$ Yanagi

\section{Abstract}

Purpose To report the therapeutic outcome of transpupillary thermotherapy (TTT) for subfoveal choroidal neovascularizarion (CNV) in brown retina using a diode-laser with the setting of lower energy level compared to the previous studies on light-pigmented Caucasian patients.

Methods A total of 19 subfoveal CNVs in 18 patients were treated with TTT. The power of diode-laser was set $160 \mathrm{~mW}$ for $1.2 \mathrm{~mm}$ beam, $270 \mathrm{~mW}$ for $2.0 \mathrm{~mm}$ beam, and $400 \mathrm{~mW}$ for $3.0 \mathrm{~mm}$ beam, and the laser was delivered for $1 \mathrm{~min}$ through a slit-lamp mounted-delivery system. Patients were followed up for a mean of 8.8 months (4-12 months). Visual acuity and the fundus change as judged by funduscopic examination and simultaneous fluorescein and indocyanine green angiography were evaluated. Visual acuity was measured by a Japanese standard Landolt visual acuity chart and converted to logarithm of the minimal angle resolution $(\log$ MAR) visual acuity for statistical analysis. Improvement or decline in vision was defined as change of more than $\mathbf{0 . 2}$ in log MAR visual acuity.

Results In eyes with minimally classic or occult only $\mathrm{CNV}$, visual acuity improved in two eyes (18\%) stabilized in seven eyes $(64 \%)$ and worsened in two eyes (18\%).

In eyes with predominantly classic $\mathrm{CNV}$, visual acuity improved in two eyes (25\%), stabilized in four eyes $(50 \%)$ and worsened in two eyes (25\%). In all, $15(84 \%)$ eyes of all studied subjects had improvement in exudation. Two (11\%) and one (5\%) eye(s) were noted to have a significant post-treatment haemorrhage and retinal pigment epithelial tear, respectively.
Conclusion In patients with brown retinal colour, the treatment outcome of TTT was comparable to that of light-pigmented Caucasian patients with approximately half the laser power energy. Further randomized control studies are warranted.

Eye (2004) 18, 615-618. doi:10.1038/sj.eye.6700733

Published online 16 January 2004

Keywords: age-related macular degeneration; transpupillary thermotherapy; retinal colour; retinal pigmentation; choroidal neovascularization

\section{Introduction}

Exudative age-related macular degeneration (AMD), characterized by choroidal neovascularization (CNV), is a major cause of visual loss in developed countries. Although laser photocoagulation treatment for subfoveal $\mathrm{CNV}$ is beneficial in selected patients, ${ }^{1}$ severe visual loss is inevitable immediately after the treatment due to the damage to the overlying retina. Treatment of $\mathrm{CNV}$ with minimum damage to the healthy retina, such as photodynamic therapy, ${ }^{2}$ has been proven to be of benefit with predominantly classic CNV. In addition, transpupillary thermotherapy (TTT). ${ }^{3,4}$ using diode-laser, is under investigation. Diode-laser TTT has advantages over the conventional laser-photocoagulation, since the laser is mainly absorbed in the choroid and theoretically enables the choroidal lesion treatment with minimum damage to the neural retina. ${ }^{5}$ TTT works by elevating the choroidal temperature by about $10^{\circ}$ and thus, inducing involution of the neovascular membrane through an indirect heat effect rather than direct
Department of

Ophthalmology University of Tokyo School of Medicine, Bunkyo-ku Tokyo, Japan

Correspondence: Y Yanagi Department of Ophthalmology University of Tokyo Schoo of Medicine, 7-3-1 Hongo Bunkyo-ku

Tokyo 113-8655, Japan Tel: + 81358008655 Fax: +81338170798 E-mail: yanagi-tky@ umin.ac.jp

Received: 7 June 2003 Accepted: 1 July 2003 Published online: 16 January 2004 
photocoagulation as does conventional thermal laser. Indeed, initial nonrandomized case studies showed that TTT might be a potential treatment for $\mathrm{CNV}$ in light-pigmented Caucasian patients. ${ }^{3,4}$ In TTT, fundus pigmentation is one of the major factors affecting the hyperthermic effect of the laser. However, to date, the outcome of this therapeutic intervention in patients with relatively pigmented (brown) retinal colour remains largely unknown. In this case series, we report the therapeutic outcome of TTT in Japanese patients with the laser setting of lower energy level compared to the previous studies.

\section{Materials and methods}

This is a retrospective, noncomparative interventional case series. All patients were treated between January 2001 and December 2001 at the outpatient clinic of the Tokyo University Hospital. Patients were diagnosed with subfoveal CNV associated with AMD according to the classification of Macular Photocoagulation Study Group. ${ }^{6}$ Cases with polypoidal choroidal vasculopathy, ${ }^{7}$ diagnosed on the basis of indocyanine green angiography finding, were excluded. In total, 19 eyes of 18 patients (17 male and one female patient(s)) with the mean patient age of 74.2 years (51-94 years) were treated. At the initial visit, all the patients underwent a general ophthalmic examination including visual acuity, slit-lamp examination and fundus examination, simultaneous fluorescein and indocyanine green angiography using scanning laser ophthalmoscope (SLO; Rodenstock, Germany). Best-corrected visual acuity was measured by Snellen's acuity and translated into logarithm of the minimal angle of resolution (log MAR). Classification of $\mathrm{CNV}$ was based on the fluorescein angiography. In all, 11 (58\%) CNVs were minimally classic or occult only and seven $(42 \%)$ CNVs were predominantly classic. Initial visual acuity was below 20/200 in nine eyes (47\%), between $20 / 200$ and $20 / 50$ in four eyes (21\%), and above $20 / 50$ in six eyes $(32 \%)$.

For TTT, Institutional Ethical committee approval was obtained and written informed consents were obtained from all the studied subjects. TTT was performed as described elsewhere ${ }^{3,4}$ with modifications. The major modification was the change in the settings of the laser power. To circumvent an excessive hyperthermic effect that could arise due to the difference in fundus pigmentation between Japanese and light-pigmented Caucasian patients, the power of the laser was set $160 \mathrm{~mW}$ for $1.2 \mathrm{~mm}$ beam, $270 \mathrm{~mW}$ for $2.0 \mathrm{~mm}$ beam, and $400 \mathrm{~mW}$ for $3.0 \mathrm{~mm}$ beam, which were approximately half the power that were used in the previous studies. ${ }^{3,4}$ Using three-mirror Goldmann's lens, diode-laser was delivered for $1 \mathrm{~min}$ through a slit-lamp mounted-delivery system. Retreatment was given to the patients who retained active CNV for more than 3 months following TTT as judged by funduscopic examination and fluorescein angiography.

Patients were followed up for a mean of 8.8 months (4-12 months). Follow-up was arranged at 2, 4, and 6 weeks and 3, 6, 9, and 12 months. At each visit, general ophthalmic examination including visual acuity, slit-lamp examination, and fundus examination was performed. Simultaneous fluorescein and indocyanine green angiographies were performed at 3, 6, 9, and 12 months after TTT. Visual acuity and fundus change as judged by fundus examination and angiographies were evaluated at 3 months after treatment and at the last visit.

Visual acuity was measured using a Japanese standard Landolt visual acuity chart at $5 \mathrm{~m}$ and converted to Snellen's acuity. For Snellen's visual acuity equivalent lower than 20/200, the patients were asked to read a Landolt visual acuity card that corresponds in size to visual acuity of $20 / 200$ at $4,3,2,1$, and $0.5 \mathrm{~m}$. For statistical analysis, visual acuity were converted into $\log$ MAR scores according to the following formula:

$$
\log M A R=-\log _{10} \text { (visual acuity fraction) }
$$

Using this conversion, each 0.1 decrement in the $\log$ MAR visual acuity scores corresponds to the loss of one line on a logarithmic visual acuity chart. Notations of counting fingers and hand motions were counted as two and three lines worse than $2 / 200$, respectively. Improvement or decline in vision was defined as change of more than 0.2 in $\log$ MAR visual acuity.

\section{Results}

Of the 19 eyes, 12 (63\%) received only one treatment session, while six (32\%) and one (5\%) eye(s) received two and three treatment sessions, respectively. At 3 months after treatment, vision improved in five eyes (16\%), stabilized in 11 eyes (58\%), and worsened in three eyes $(16 \%)$. At the last visit, vision improved in four eyes $(21 \%)$, stabilized in 11 eyes $(58 \%)$, and worsened in four eyes $(21 \%)$ of all the studied subjects (Figure 1). In eyes with minimally classic or occult only CNV, vision improved in two eyes (18\%), stabilized in seven eyes $(64 \%)$, and worsened in two eyes (18\%). In eyes with predominantly classic $\mathrm{CNV}$, visual acuity improved in two eyes (25\%), stabilized in four eyes $(50 \%)$, and worsened in two eyes (25\%).

In all, 15 (84\%) eyes of all the studied subjects had improvement in exudation (Figure 2). Two (11\%) and one $(5 \%)$ eye(s) were noted to have a significant post-treatment haemorrhage (Figure 3) and retinal pigment epithelial tear (Figure 4), respectively. 


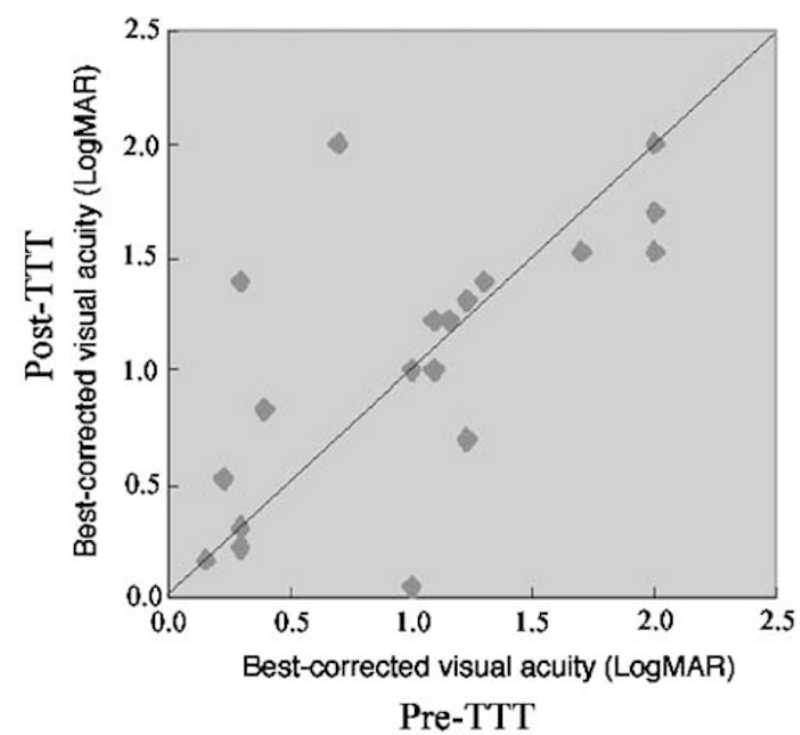

Figure 1 Visual acuity before and after TTT.
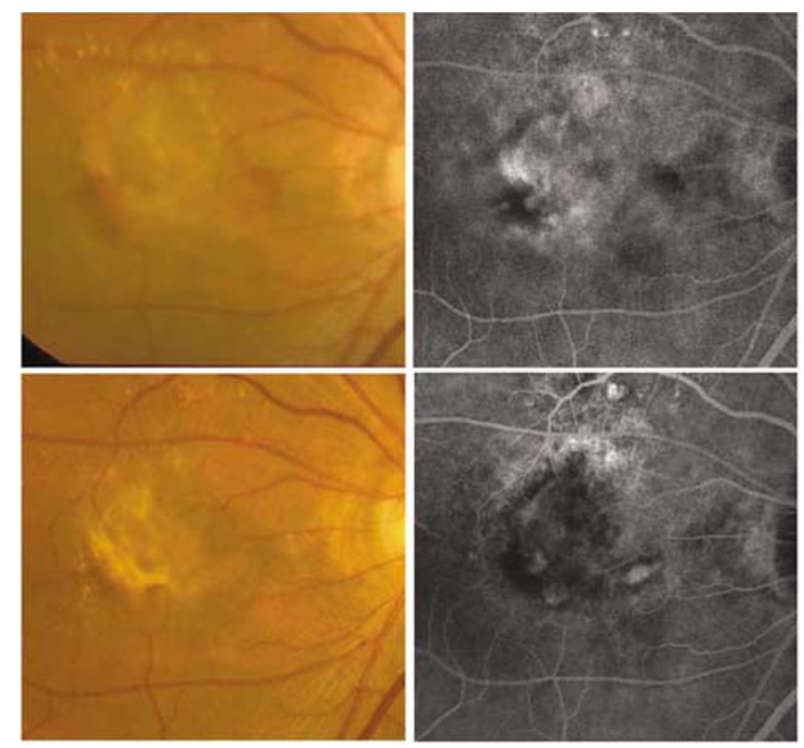

Figure 2 Color (top left) and fluorescein (top right) image showing a subfoveal CNV. Color (bottom left) and fluorescein (bottom right) image showing a resolution of $\mathrm{CNV}$ and improvement in exudation following TTT.

\section{Discussion}

TTT uses hyperthermic effect to disrupt the choroidal neovascular membrane. ${ }^{5}$ Fundus pigmentation affects retinal irradiance and subsequent retinal temperature rise. Thus, to exert therapeutic effect, the laser power must be set depending on retinal colour. In this study, we employed the power approximately half compared with the previous studies on light-pigmented Caucasian patients. ${ }^{3,4}$ Visual acuity stabilized or improved in $79 \%$ of all the studied subjects under our condition, similar to the previous studies, which demonstrated stabilization or improvement of visual acuity in 78 and $75 \%$ of the patients after a mean follow-up of 6.1 and 13 months respectively. ${ }^{3,4}$ It might not be compared because of the difference in baseline characteristics; however, it seems likely that the level of the laser power was not too low for our patients because our result demonstrated a high closure rate $(84 \%)$.

Effect of diode-laser on the neural retina and retinal pigment epithelium is minimum below its photocoagulation threshold, but it can damage them with excessive laser power. Whitening of the lesion can be observed presumably when the retinal pigment epithelium and/or neural retina are damaged. Previous investigators chose to reduce the laser power when such phenomenon was observed to avoid excessive hyperthermic effect. ${ }^{3,4,9}$ In our case series, such retinal change attributable to excessive hyperthermic effect was not observed during treatment, suggesting that our condition was not excessive compared to the studies. In addition, a previous report has documented spot-size-related post-TTT chorioretinal atrophy especially in patients dark-haired in their youth and suggested that laser-power settings should be progressively diminished with increasing patient pigmentation. ${ }^{10}$ In our case series, no such complication was observed, supporting that our laser setting was not excessive compared to the study. However, significant post-treatment haemorrhage and retinal pigment epithelial tear, complications also reported previously, $3,4,11$ were observed in some patients. These complications might be more related to the underlying disease process rather than the specific treatment. Alternatively, it is possible that the laserpower setting was inappropriate for these patients and that appropriate laser-power setting must be determined empirically to avoid such complications. Probably, TTT with lower laser energy might be appropriate for certain set of patients or certain group of patients might not be eligible for TTT at all.

Although our study is retrospective, includes small sample and limited follow-up time and cannot address whether TTT is effective for CNV treatment, this has demonstrated encouraging results that TTT may show promise in treating not only occult CNV but also classic CNV in brown retina. To clarify whether TTT is effective for CNV treatment on patients with brown retina and if so, to determine the eligible patient group and the appropriate laser-power setting, further prospective randomized control studies are warranted. 


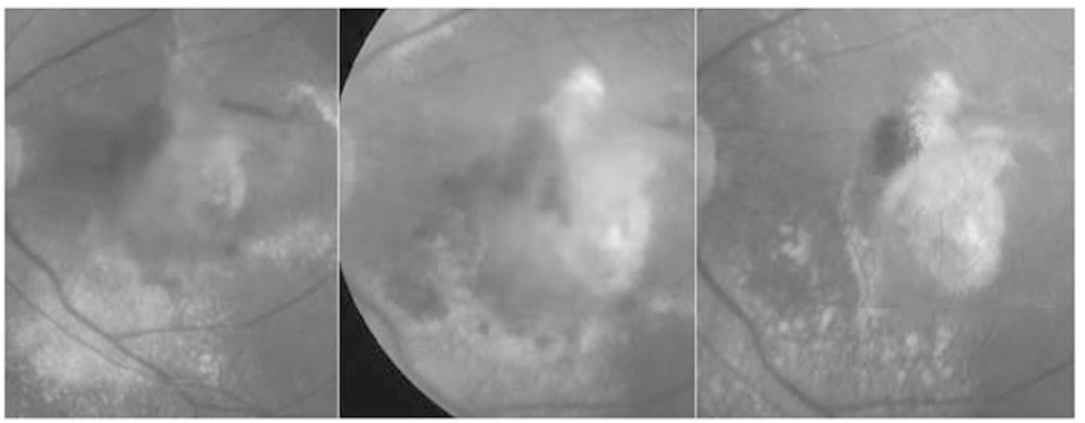

Figure 3 Fundus photographs showing significant haemorrhage after TTT. Left panel shows the fundus image before TTT. Middle and right panels show the fundus images 1 week and 1 month after TTT, respectively.

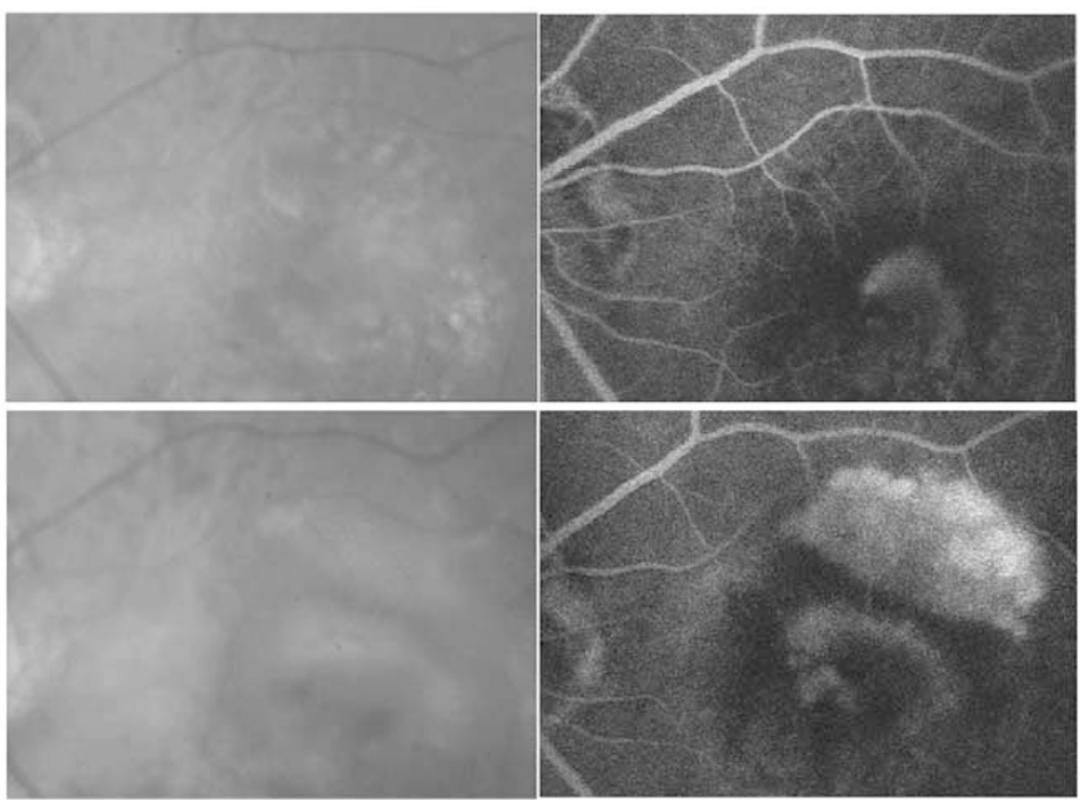

Figure 4 Fundus photographs (left panels) and fluorescein images (right panels) of retinal pigment epithelial tear after TTT.

\section{References}

1 Macular Photocoagulation Study Group. Laser photocoagulation of subfoveal neovascular lesions of agerelated macular degeneration. Updated findings from two clinical trials. Arch Ophthalmol 1993; 111: 1200-1209.

2 Bressler NM. Photodynamic therapy of subfoveal choroidal neovascularization in age-related macular degeneration with verteporfin: two-year results of 2 randomized clinical trials-tap report 2. Arch Ophthalmol 2001; 119: 198-207.

3 Reichel E, Berrocal AM, Ip M, Kroll AJ, Desai V, Duker JS et al. Transpupillary thermotherapy of occult subfoveal choroidal neovascularization in patients with age-related macular degeneration. Ophthalmology 1999; 106: 1908-1914.

4 Newsom RS, McAlister JC, Saeed M, McHugh JD. Transpupillary thermotherapy (TTT) for the treatment of choroidal neovascularisation. Br J Ophthalmol 2001; 85: 173-178.

5 Rogers AH, Reichel E. Transpupillary thermotherapy of subfoveal occult choroidal neovascularization. Curr Opin Ophthalmol 2001; 12: 212-215.

6 Macular Photocoagulation Study Group. Subfoveal neovascular lesions in age-related macular degeneration.
Guidelines for evaluation and treatment in the macular photocoagulation study. Arch Ophthalmol 1991; 109: 1242-1257.

7 Yannuzzi LA, Sorenson J, Spaide RF, Lipson B. Idiopathic polypoidal choroidal vasculopathy (IPCV). Retina 1990; 10: 1-8.

8 Spaide RF, Yannuzzi LA, Slakter JS, Sorenson J, Orlach DA. Indocyanine green videoangiography of idiopathic polypoidal choroidal vasculopathy. Retina 1995; 15: $100-110$

9 Lanzetta P, Michieletto P, Pirracchio A, Bandello F. Early vascular changes induced by transpupillary thermotherapy of choroidal neovascularization. Ophthalmology 2002; 109: 1098-1104.

10 Auer C, Tao Tran V, Herbort C. Tranpupillary thermotherapy for occult subfoveal neovessels in agerelated macular degeneration: importance of patient pigmentation for the determination of laser settings. Klin Monatsbl Augenheilkd 2002; 219: 250-253.

11 Thompson JT. Retinal pigment epithelial tear after transpupillary thermotherapy for choroidal neovascularization. Am J Ophthalmol 2001; 131: 662-664. 